\title{
The Effects of Segmentation-Based Shadow Removal on Across-Date Settlement Type Classification of Panchromatic QuickBird Images
}

\author{
F. P. S. Luus, F. van den Bergh, and B. T. J. Maharaj
}

\begin{abstract}
Settlement classifiers for multitemporal satellite image analysis have to overcome numerous difficulties related to across-date variances in viewing- and illumination geometry. Shadow anisotropy is a prominent contributing factor in classifier inaccuracy, so methods are introduced in this study to enable minimum-supervision classifier design that mitigate the effects of shadow profile differences. A segmentation-based shadow detector is proposed that utilises a panchromatic segment merging algorithm with parameters that are robust against dynamic range variances seen in multitemporal imagery. The proposed shadow detector improves on the settlement classification accuracy achieved by fixed threshold detection paired with shadow removal in the presented case-study. The relationship between shadow detection accuracy and settlement classification accuracy is investigated, and it is shown that shadow removal produces greater settlement accuracy improvements for acrossdate experiments specifically.
\end{abstract}

Index Terms-Image texture analysis, image segmentation, feature extraction, urban areas, remote sensing.

\section{INTRODUCTION}

$\mathbf{M}$ ULTITEMPORAL satellite data includes imagery from different dates and times such that varying viewingand illumination geometry manifests across the images due to the different acquisition modes and conditions. These differences must be accounted for when doing minimal-supervision settlement type classification, as it can severely degrade classification accuracy. A primary assumption in pattern classification is that of relative feature constancy, but in remote sensing this feature invariance is not guaranteed due to the varying nature of satellite-borne image acquisition.

The high resolution QuickBird satellite has a 30 degree offnadir wide accessible ground swath, which gives it the ability to acquire images from very different azimuth angles. So images of the same area may look completely different in some respects due to the viewing geometry variances. In addition, the seasonality and time of day during acquisition determine the sun elevation and solar illumination angle of the landscape. This may produce bidirectional spectral reflectance effects

F. P. S. Luus and B. T. J. Maharaj are with the Department of Electrical, Electronic and Computer Engineering, University of Pretoria, Pretoria, South Africa.

F. van den Bergh is with the Remote Sensing Research Unit, Meraka Institute, Council for Scientic and Industrial Research, Pretoria, South Africa.

Address correspondence to F.P.S. Luus, Department of Electrical, Electronic and Computer Engineering, University of Pretoria, Pretoria, South Africa. Email: luus@ieee.org. Telephone: +27 721381735.

Color versions of one or more of the figures in this paper are available online at http://ieeexplore.ieee.org. and varying shadow profiles across multitemporal imagery. Shadowing is an example of one of the illumination effects that presents with more adverse variance in multitemporal imagery, but it is that well defined presentation that makes it possible to do shadow detection and masking for calculated feature invariance.

There are strong directional variations in urban surface features [1] potentially resulting in very different viewingand illumination geometries in multitemporal imagery. To accurately model viewing- and illumination geometry, detailed augmenting information such as digital surface models and multi-angle imaging data is a requirement. Topographic correction of surface reflectance was done by $\mathrm{Li}$ et al. [2], and terrain illumination correction was performed by $\mathrm{Wu}$ et al. [3] with shadow and occlusion detection using digital surface models. The viewing angle effects on spectral response and discrimination of urban land-cover types were investigated with multi-angle spectroradiometer data by Huang et al. [4].

Working with such augmenting information is troublesome, as data availability is a prime concern and a further dimension of complexity is added to the analysis. The approach outlined in this research is based on the high resolution digital imagery product only, without the need for augmenting metadata. The onus is thus on the efficacy of the artificial intelligence used to extract accurate information from imagery, regardless of surface and shading anisotropy.

Pattern classification relies on differential measures characterizing the distinct natures of every class, and texture features have been found [5] to be an appropriate measure of settlement type patterns. The texture-based approach will be extended in this study, with consideration toward illumination geometry invariance. By removing shadows a major component of illumination variance can be addressed. Shadow detection is the initial step in the shadow removal process, and fixed intensity thresholding is the dominant panchromatic detection method found in the literature [6].

Most shadow detection techniques are based on the rich information provided by multispectral imagery, especially the shadow property of maintained relative color but reduced intensity (retinex theory) [7]-[9]. A morphological shadow index, based on the spectral-structural characteristics of shadows, was proposed by Huang and Zhang [10] to automatically indicate the presence of shadows in high-resolution imagery through the local extraction of dark structures within a range of sizes in different scales and directions.

Accurate multispectral shadow detection was performed by 
Makarau et al. [11] in a supervised setting by modeling the relationship between direct sunlight and scattered light using a blackbody radiator model. Digital surface models were also used to perform a line-of-sight analysis with solar position and elevation to determine the shadow profile [12]. There is a lack of sophisticated panchromatic-only shadow detectors in the literature. Effective intensity thresholding was used by Wei et al. [6] by choosing the lowest intensity class from unsupervised clustering by histogram-peak selection.

Panchromatic shadow detection is a prime focus in this work, in order to supplement the thin base of available methods. Unsupervised panchromatic segmentation with a novel merging algorithm is proposed in this research, with the aim of post-segmentation shadow detection through both thresholdbased and supervised object selection. For the novel segmentation algorithm a simple graph cut method is described, which requires only panchromatic information and does not require markers or segment seeds to maintain linear computational complexity in the number of image pixels [13]. The graph cut algorithm is bespoke for high-resolution panchromatic segmentation and it uses the localized dynamic range to actively reduce the merging threshold in a way that preserves fine structural detail.

An object-based panchromatic shadow detector is contributed, which does supervised selection of shadow objects based upon panchromatic-only properties such as the standard deviation and mean of the object segment intensity. The supervised detector is compared against an unsupervised objectbased shadow detector that classifies segments with intensities less than a fixed threshold as shadow. The comparison is performed in terms of both shadow detection accuracy and settlement classification accuracy with shadow removal.

The second step of shadow removal is to either do shadow correction or shadow masking. Gamma correction, linearcorrelation correction and histogram matching are popular shadow correction techniques. Shadow detection using fixed thresholding on a multispectral object-segmented image was done by Liu and Yamazaki [14], and linear-correlation shadow correction was then performed on the shadow areas. Basic shadow correction using global histogram matching is compared in this study against fine correction that relies on region growing and localised histogram matching.

Shadow masking removes shadow areas from the texture feature calculations without the need for image correction. The settlement classification performance with shadow masking under fixed thresholding detection and shadow segmentation is also investigated. Shadow removal with detection based on fixed intensity thresholding was previously studied by the authors [15], and the research is extended here to include segmentation-based methods for improved shadow detection.

The primary objective of the main experiment is to determine the efficacy of shadow removal in improving across-date settlement classification accuracy, and to investigate the relationship between shadow detection accuracy and settlement classification accuracy improvements. The experimental input includes at least two high-resolution panchromatic images of the same area without metadata, acquired on different dates with significant shadow profile differences, and a small set of ground truth shadow masks for the image of each date. The ground truth shadow masks cover part of each settlement type to give a fully representative shadow mask sample to the supervised segment-based shadow detector.

Shadow detection comparisons are performed between a fixed threshold pixel-based detector, an unsupervised segmentbased detector, and a supervised segment-based detector. The shadow removal methods that are compared include shadow masking, basic shadow correction and fine shadow correction. For settlement classification two texture features are compared, namely LBP and GLCM features. The novel contributions of the study include the panchromatic segmentation-merging algorithm, the unsupervised and supervised segmentation-based shadow detectors, and the statistical analysis of the relationship between shadow detection accuracy and settlement classification accuracy improvements.

The methodology of the settlement type classification experiment is outlined in Section II, and the shadow detection algorithms and shadow-mitigation methods are explained in Sections III and IV, respectively. A description of the study area is given in Section $\mathrm{V}$ and the exact experimental setup is defined in Section VI. The results of the settlement classification and shadow detection accuracy experiments are given and interpreted in Section VII, and concluding remarks are shared in Section VIII.

\section{Methodology}

The effects of varying shadow profiles on across-date settlement classification accuracy are determined by removing shadows before calculating the settlement features. The approach to across-date shadow removal is discussed in the next two sections, where specifics on the implementation of shadow detection and removal are given. An outline of the modified feature calculations is depicted in Figure 1.

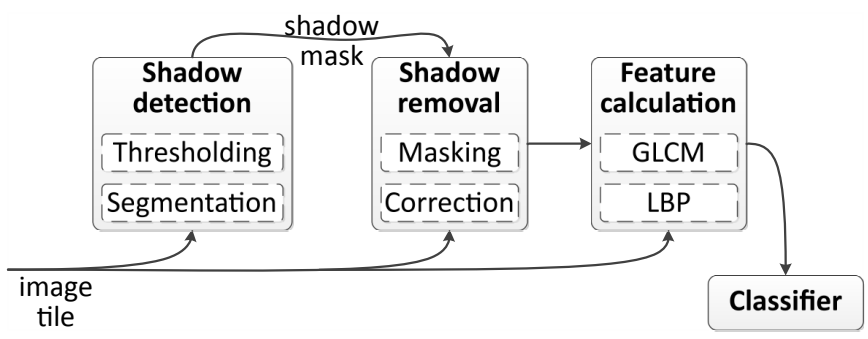

Fig. 1. The shadow invariant approach to settlement feature calculation.

Gray-level co-occurrence matrix (GLCM) [16] and local binary pattern (LBP) [17] texture features have been shown to be the best performing features for settlement type classification [5]. Texture features are sensitive to spurious differences in viewing- and illumination geometry [18], hence the decision to investigate the effects of shadow removal with the use of GLCM and LBP features in this work.

To characterise the effect of differing shadow profiles on across-date classification accuracy, a comparison is made with shadows intact and with shadows removed. For statistically significant improvements in accuracy the hypothesis is proposed that shadow differences cause significant across-date differences in texture features of the same settlement class. 


\section{Shadow Detection}

Shadow areas caused by the occlusion of sunlight have to be identified before shadow-effect mitigation methods may be applied. A literature review on object-based shadow detection and segmentation-merging methods is conducted in this section to serve as background for the segmentation-based shadow detection algorithm design. A basic approach to panchromatic segmentation is described and it is then shown how a refined merging algorithm can improve the segmentation result. The segmentation algorithm is then employed in a shadow detector, followed by a review of fixed threshold pixel-based detection which is used as benchmark.

\section{A. Object-based shadow detection}

Object-based shadow detection is thus refined in this study for suitable use with the limited information provided by panchromatic-only imagery. Object-based image analysis splits an image into objects based on heterogeneity criteria, which yield the dual advantages of region-based methods, namely a reduction in the number of segments and that those primitives carry more information. Segmentation is conducted by progressively merging neighbouring primitives according to a given predicate, until a homogeneity criterion is met for all resulting segments.

Region-merging algorithms and multiscale image segmentation algorithms are popular foundational procedures of objectbased image analysis in which homogeneous image object primitives are obtained [19]. Region-merging algorithms have been based on spatio-temporal similarity [20], statistical properties [21], [22], and graph properties [23], [24]. The merging algorithms proposed by Peng et al. [25] and Sun et al. [26] rely on colour information and semi-supervised methods to perform multispectral object segmentation.

The different cut criteria in graph theory that have been proposed for merging include the minimum cut [27], normalized cut [28], and the ratio cut [29]. The graph cut methods produce a desired segmentation through the global optimization of a cost function, but the optimization processes are often computationally inefficient for the large data of remote sensing [30]. In this study a fast graph-based merging algorithm is proposed that gives robust segmentations in spite of the dynamic range variations seen in multi-date imagery.

Multiscale segmentation algorithms have been used in remote sensing image analysis, including watershed segmentation, multiresolution segmentation [31], hierarchical segmentation [32], and mean-shift segmentation [33]. Huang and Zhang [34] proposed an adaptive mean-shift segmentation algorithm for clustering remote sensing images in both spatial and spectral domains with an adaptively chosen bandwidth. A salient factor in multiscale segmentation algorithms is the determination of an appropriate merging scale, which influences the amount of over- or under-segmentation that results. The choice of the scale parameter is difficult as it depends on the underlying data and the desired granularity for the specific application. This issue is addressed in this work by reducing the sensitivity of the segmentation on the scale or merging parameter during region-merging.
Watershed segmentation is an efficient first step for objectbased shadow detection, but an over-segmentation is normally produced where merging is required to reduce the number of segments for an accurate account of the image objects. In the case of the merging of a watershed segmentation, compensation is required to enforce finer structural detail by preventing bleed resulting from a graph cut merging algorithm used by Li et al. [35] as well as Pun and An [36]. This complex segmentation should be done in the absence of markers, to eliminate the marker density parameter [37], which is hard to control without a priori information for settlements with varying characteristics.

\section{B. Basic segmentation}

The input image matrix $I$ is filtered with horizontal and vertical Sobel edge-emphasizing filters producing two cardinal gradient images $I_{x}$ and $I_{y}$, respectively. A final gradient magnitude image is then calculated as $I_{g}=\sqrt{I_{x} \circ I_{x}+I_{y} \circ I_{y}}$, where the entry-wise Hadamard product is denoted by $\circ$. Topographic distance is used to obtain an initial watershed segmentation from the gradient magnitude image $I_{g}$, according to the algorithm of Meyer [38] with 8-connected neighbourhoods. An example of the initial segmentation obtained with the watershed algorithm is shown for different dates $d_{1}$ and $d_{2}$ in Figure 2.

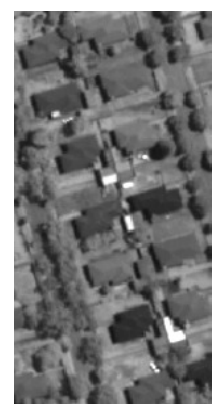

(a) $d_{1}$-image

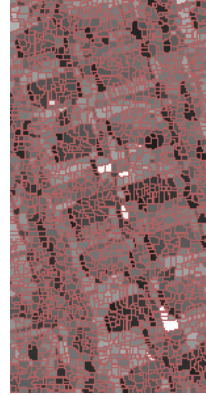

(b) $d_{1}$-watershed

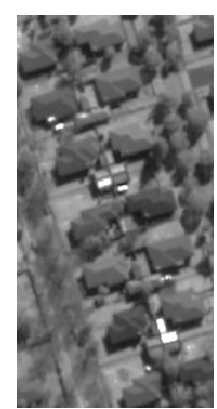

(c) $d_{2}$-image

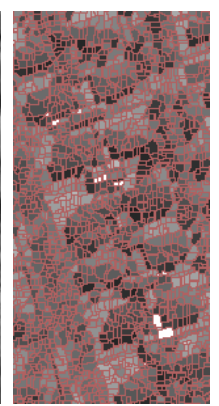

(d) $d_{2}$-watershed
Fig. 2. Watershed segmentation of two images of the same formal settlement acquired on different dates $d_{1}$ and $d_{2}$.

The watershed segmentation reduces the number of graph components involved in the calculation of the final segmentation, but a further merging of the watershed segments is required due to a typical over-segmentation produced by the watershed algorithm. As with multiscale segmentation algorithms [31], [32] a merging parameter is used in this study to dictate the amount of over-segmentation after merging. The basic segmentation discussed in this part of the paper is later contrasted with a more robust segmentation that uses a secondary merging parameter to reduce the sensitivity of the segmentation to the primary merging parameter.

Let the $N_{o}$ watershed segments be represented by vertices $n_{1}, n_{2}, \cdots, n_{N_{o}}$ of a directed graph $\mathcal{G}$, which will be used by the merging algorithm. For each vertex $n_{i}$ the graph forms a directed edge $l_{i j}$ to each 8-connected neighbouring segment $n_{j}$ (from neighbourhood set $\mathcal{N}\left(n_{i}\right)$ ) that shares a segment border with $n_{i}$. Let the mean pixel intensity of a segment $n_{i}$ be given by $\mu_{Y}\left(n_{i}\right)$, then a simple merging 
criterion is $\left|\mu_{Y}\left(l_{i j}\right)\right|=\left|\mu_{Y}\left(n_{i}\right)-\mu_{Y}\left(n_{j}\right)\right| \leq \alpha(\lambda)$. When the merging criterion is met for an edge $l_{i j}$ then the segments $n_{i}$ and $n_{j}$ should be combined. Here $\alpha(\lambda)=\lambda Y_{\max }$ is an intensity difference threshold calculated in terms of a merging parameter $\lambda$ and the maximum intensity $Y_{\max }$ as determined by the image bit-depth. The merging parameter $\lambda$ determines the amount of under- or over-segmentation and in that sense a parallel is drawn to the scale parameter as found in multiscale segmentation algorithms.

By removing edges with $\left|\mu_{Y}\left(l_{i j}\right)\right|>\alpha(\lambda)$ a potentially reduced graph $\mathcal{G}^{*}$ is obtained, which may then consist of several smaller graphs $\mathcal{G}_{i}^{*}$ that represent the remaining merged segments. The vertices of a subgraph $\mathcal{G}_{i}^{*}$ are to be combined into a single segment $n_{i}^{*}$ to form part of the merged segmentation. For a small difference threshold and merging parameter of $\lambda=0.039$ a still over-segmented merge is obtained in Figure 3. In Figures 3, 4 and 6 the merged segment borders are overlayed as red lines in $d_{1}$-merged and $d_{2}$-merged, and every segment is replaced by its mean pixel intensity in $d_{1}$-avg and $d_{2}$-avg.

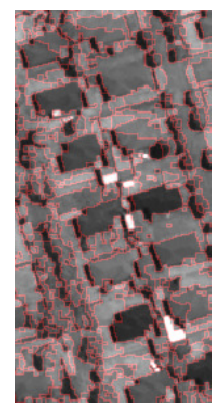

(a) $d_{1}$-merged

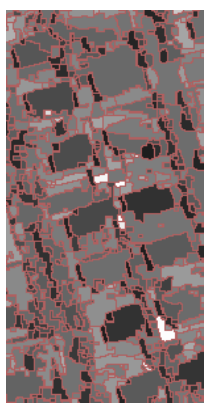

(b) $d_{1}$-avg

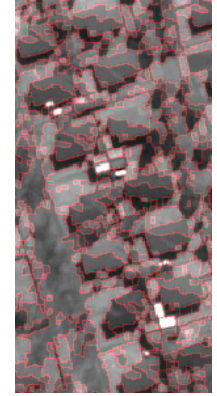

(c) $d_{2}$-merged

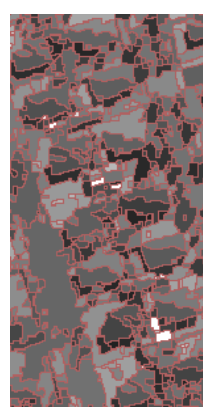

(d) $d_{2}-\operatorname{avg}$
Fig. 3. Basic merging with a merging parameter of $\lambda=0.039$.

Doubling the difference threshold with a merging parameter of $\lambda=0.078$ produces a less over-segmented merge for $d_{1}$ as shown in Figure 4, but there is a large discrepancy which results in a more under-segmented merging for $d_{2}$. This is caused by a difference in the dynamic range of the acrossdate images due to varying acquisition modes, illumination differences determined by the solar illumination angle and sun elevation, and the effects of histogram matching.

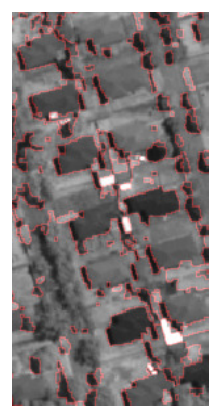

(a) $d_{1}$-merged

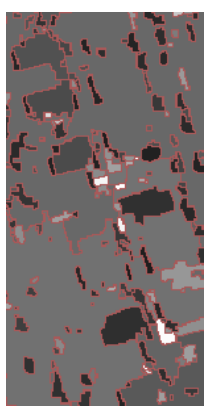

(b) $d_{1}$-avg

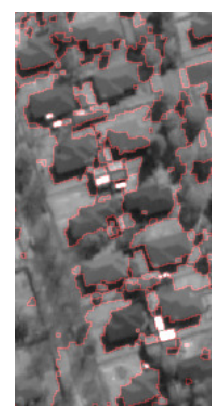

(c) $d_{2}$-merged

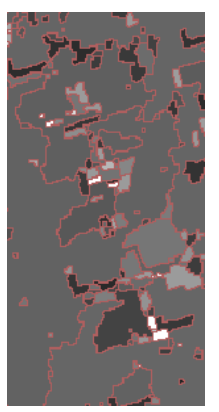

(d) $d_{2}$-avg
Fig. 4. Basic merging with a larger merging parameter of $\lambda=0.078$.

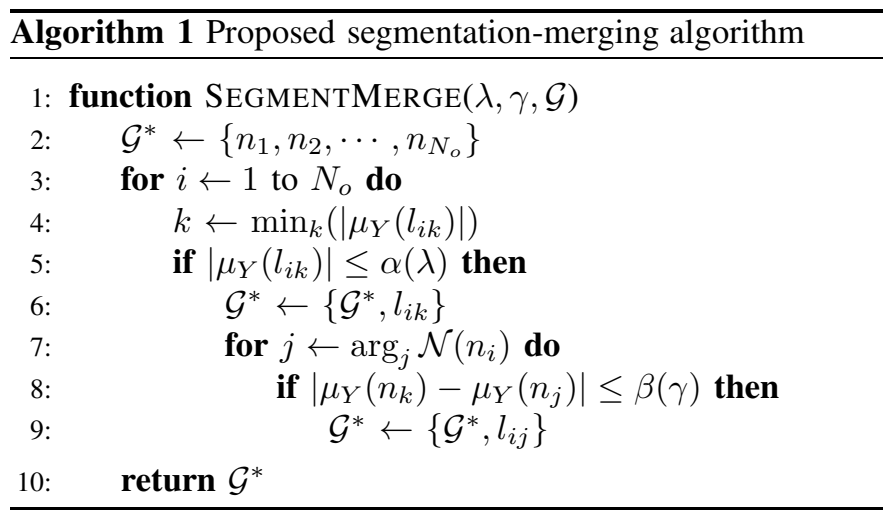

\section{Proposed merging algorithm}

A further difference threshold $\beta(\gamma)=\gamma Y_{\max }$ and a secondary merging parameter $\gamma$ is introduced in the proposed merging algorithm to reduce the $\alpha$-sensitivity of the resultant segmentation. The effect of the difference threshold $\alpha(\lambda)$ can be reduced by selecting only the neighbour $n_{k}$ with the smallest intensity difference for inclusion in the merged segment, based on the $\alpha$-criterion. If a remaining neighbour $n_{j}$ has an intensity close to that of the smallest differing neighbour $n_{k}$, i.e. when $\left|\mu_{Y}\left(n_{k}\right)-\mu_{Y}\left(n_{j}\right)\right| \leq \beta(\gamma)$, then $n_{j}$ will be merged with the base segment $n_{i}$. The proposed merging algorithm is outlined in Algorithm 1.

A poor choice for $\lambda$ will then have a lesser effect on the final merging, especially when the smallest difference between the smallest differing neighbour $n_{k}$ and the base segment $n_{i}$ is less than a proper threshold $\alpha\left(\lambda_{\text {good }}\right)$. In that instance of $\left|\mu_{Y}\left(l_{i k}\right)\right| \leq \alpha\left(\lambda_{\text {good }}\right)$ the difference threshold $\alpha(\lambda)$ is effectively replaced by $\alpha\left(\lambda_{\text {good }}\right)$ and the quality of the merging then depends on the secondary threshold $\beta(\gamma)$. An example of a reduced graph $\mathcal{G}^{*}$ is shown in Figure 5a), which consists of $N_{m}$ disconnected subgraphs $\mathcal{G}_{1}^{*}, \mathcal{G}_{2}^{*}, \cdots, \mathcal{G}_{N_{m}}^{*}$ that form the merged segments $n_{1}^{*}, n_{2}^{*}, \cdots, n_{N_{m}}^{*}$ as shown in Figure $5 \mathrm{~b}$ ). The merged segments are counted as vertices $n_{i}^{*}$ of the resultant merged graph $\mathcal{G}_{m}$. In Figure 5a) the green and blue edges met the $\alpha$-criterion and $\beta$-criterion, respectively, and are retained in the reduced graph.

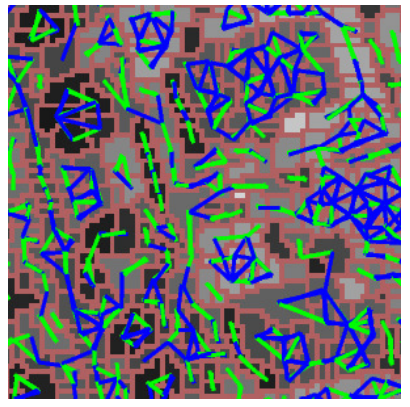

(a) reduced graph

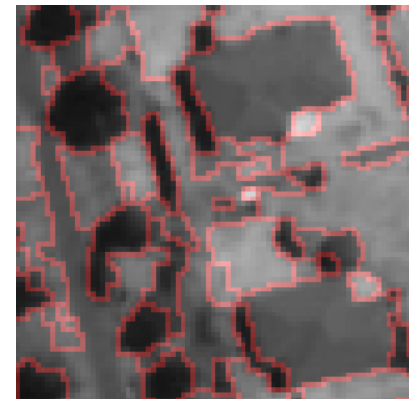

(b) merged segmentation
Fig. 5. The reduced graph $\mathcal{G}^{*}$ and the corresponding merging with the proposed algorithm with $\lambda=0.078$ and $\gamma=0.039$.

The efficacy of the proposed merging algorithm with secondary threshold $\beta(\gamma)$ is made clear in the comparison between Figures 4 and 6, where the same primary constraint 
$\alpha(\lambda)=\lambda Y_{\max }$ with $\lambda=0.078$ is used. The proposed algorithm produces an acceptable merging for both dates with no notable over-segmentation or under-segmentation, especially in the shadow areas. The secondary constraint of $\beta(\gamma)=\gamma Y_{\max }$ with $\gamma=0.039$ and the difference ordering of the connected edges in the proposed algorithm make the exact choice of $\lambda$ less critical.

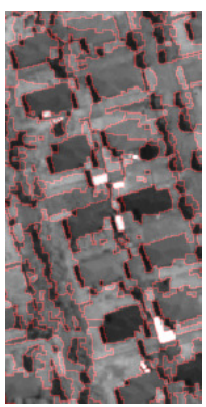

(a) $d_{1}$-merged

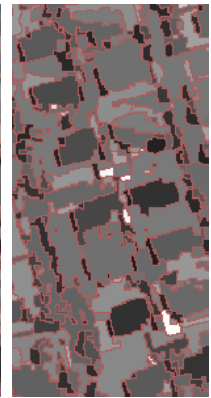

(b) $d_{1}-\mathrm{avg}$

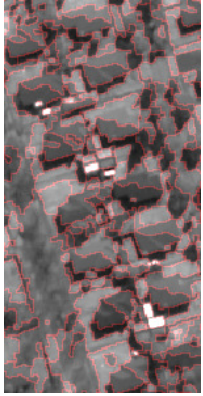

(c) $d_{2}$-merged

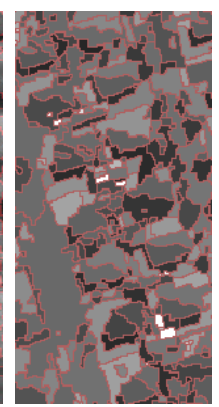

(d) $d_{2}$-avg
Fig. 6. The proposed merging with $\lambda=0.078$ and $\gamma=0.039$.

The selection of merging parameters and thresholds is still an intractable problem [32], made difficult by the fact that the segmentation purpose dictates the desired segmentation result. While an assisted selection of the values of $\lambda$ and $\gamma$ is thus still required in this work, a method is contributed to reduce sensitivity of the segmentation result to the specific choice. This is achieved through a direct query of the localized dynamic range, which is used to reduce the primary merging threshold. Fine detail can then be preserved by preventing possible bleeding due to a merging threshold that may be too large for the scene.

\section{Segmentation-based detection}

In object-based image analysis the extracted objects are characterised by distinctive features, and in the case of the merged panchromatic segmentation the mean pixel intensity $\mu_{Y}\left(n_{i}^{*}\right)$ and standard intensity deviation $\sigma_{Y}\left(n_{i}^{*}\right)$ of every segment $n_{i}^{*}$ are used as basic features. Basic segmentationbased shadow detection would then declare a segment $n_{i}^{*}$ as shadow when $\mu_{Y}\left(n_{i}^{*}\right) \leq \xi Y_{\max }$. This is used as a benchmark to evaluate a supervised segment-selection approach against. According to the low-intensity property of shadows an intensity ratio of $\xi=0.2$ has been used to decide whether a segment belongs to a shadow area. This value has been found to produce good results for the considered dataset, since it is a choice rooted in principle shadow fundamentals.

Given a binary ground truth shadow mask, a segment is classified as shadow when at least half of the pixels in the segment belong to a ground truth shadow area. Then a classifier may be trained for supervised detection using features $\mu_{Y}\left(n_{i}^{*}\right)$ and $\sigma_{Y}\left(n_{i}^{*}\right)$ with the corresponding binary ground truth classification for a segment $n_{i}^{*}$. Specifically, a multi-layer perceptron (momentum rate of 0.2 , learning rate of 0.3 and 500 training epochs) is trained with a small set of ground truth shadow masks covering every date and settlement type. The perceptron has two input nodes, one for each basic shadow segment feature, and two output units for the binary shadow classification. A single hidden layer is used with two units and unipolar sigmoid activation functions are employed in the network.

The supervised detector then comprises the proposed segmentation algorithm, a merged segment feature calculator and a trained classifier. A new image may be segmented and segment features can then be classified as non-shadow/shadow by the classifier. Examples of shadows detected by this supervised detector are shown in Figure 7 for the three main settlement types discussed in Section V. The difference in the shadow profiles of $d_{1}$ and $d_{2}$ are significant, primarily due to the seasonal variation of the two acquisitions.

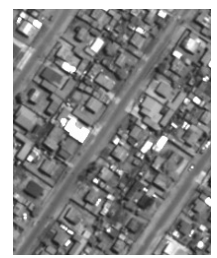

(a) FSB : $d_{1}$

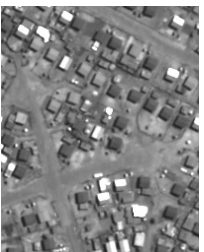

(e) FS : $d_{1}$

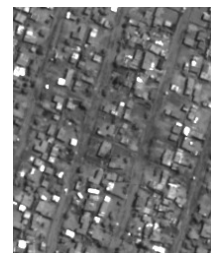

(i) OIS : $d_{1}$

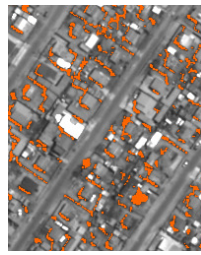

(b) $d_{1}$-shadow

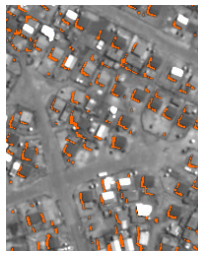

(f) $d_{1}$-shadow

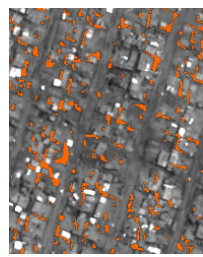

(j) $d_{1}$-shadow

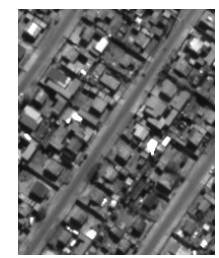

(c) FSB : $d_{2}$

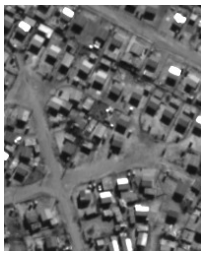

(g) FS : $d_{2}$

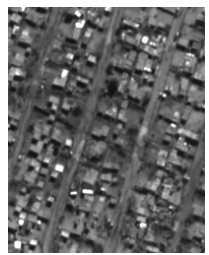

(k) OIS : $d_{2}$

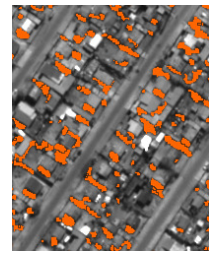

(d) $d_{2}$-shadow

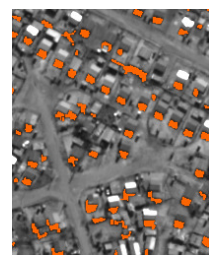

(h) $d_{2}$-shadow

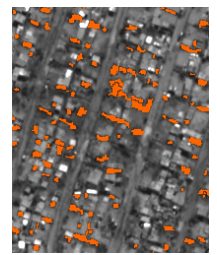

(1) $d_{2}$-shadow
Fig. 7. Supervised shadow detection examples (shown in orange) paired with original images for the three main settlement classes FSB, FS, and OIS.

A prominent weakness of segment-based detection is a higher omission error rate due to entire segments being wrongly classified as non-shadow, when a threshold-based detector would commit at least part of the segment to the shadow mask. This omission is visible in Figure 7 where some shadow areas remain undetected with the segment-based shadow detector. When part of a lighter structure falls within shadow area it is likely to be classified as non-shadow due to the higher segment intensity. This situation has a greater probability of occurring as the building heights increase and the shadows become long enough to block sunlight that would have otherwise illumed adjacent structures.

The prevalence of high-reflection surfaces in the image will influence the shadow ommission rate, and in general a larger intensity variance within the shadow areas will cause greater confusion during shadow detection. While the proposed shadow detector performs well with the smaller structures of urban areas, a refined approach will be required to accurately handle the shadows of medium- and high-rise 
buildings. The merging algorithm may be maintained, but additional intelligence is needed to decide whether parts of a structure falls within the shadow of another structure or not.

The shadow detector has a linear computational complexity in the number of image pixels, but the exact complexity is dependent on the image content, which dictates the complexity of the segmentation computation. An unoptimized Matlab implementation of the supervised segment-based shadow detector maintained an average computational speed of 8000 pixels per second per thread on a $2.8 \mathrm{GHz}$ Intel i7 computer for all data set polygons for both dates.

\section{E. Fixed thresholding pixel-based shadow detection}

A rudimentary shadow mask is obtained with a global intensity threshold to serve as a benchmark to measure the proposed segmentation-based detector against in this study. Multi-date images are histogram matched before a common fixed intensity threshold is used to classify pixels with lesser intensity as shadow. The accuracy of the threshold-based shadow masks depends on the specified threshold, therefore a range of experiments are performed over a varying threshold interval of $0 \%$ to $40 \%$ of the maximum intensity value as determined by the image bit-depth. Shadow intensities may vary in different parts of the same scene and a shadow area may form a gradient with intensities both less than and exceeding the fixed threshold. These limitations of fixed thresholding lead to shadow classification inaccuracies and may be addressed with an object-based approach involving segmentation.

\section{Shadow Removal}

A shadow mask is provided by the shadow detector, and in this study two approaches for reducing the impact of shadows on settlement type features are investigated, namely shadow masking and correction.

\section{A. Shadow masking}

Modified texture feature calculations are performed with shadow masking, where pixels belonging to a shadow area are ignored during feature determination. For GLCM calculations each pixel pair is skipped when one of its pixels falls within the shadow mask, effectively removing some off-diagonal entries in the co-occurrence matrix that would have resulted if the shadows were included. The shadow masking variant for LBP features are similarly obtained by avoiding pattern placement in locations where the central pixel would fall within the detected shadow mask.

\section{B. Shadow correction}

Shadow correction modifies the input images before feature calculation, so no adaptation of the GLCM and LBP algorithms is required in the case of correction. Basic shadow correction adds to every shadow pixel the intensity difference between the global intensity mean $\mu_{Y}(\mathcal{G})$ and the corresponding shadow region intensity mean $\mu_{Y}\left(n_{i}^{*}\right)$. The two-pixel wide transition edge outside each shadow region is then locally median filtered in a five-pixel window [39] to suppress the transition gradient.

Apart from basic correction a fine shadow correction algorithm is also investigated that takes the most frequently occurring intensity value in the two-pixel wide edge outside of the transition edge instead of the global mean when performing the histogram matching. Here the intensity difference between this outer edge intensity value and the corresponding shadow region intensity mean $\mu_{Y}\left(n_{i}^{*}\right)$ is added to each shadow pixel. The transition median filtering is also performed after this histogram matching as with the basic shadow correction. The fine shadow correction blends the lifted shadows better than with basic correction and it addresses the situation where shadows are surrounded by surfaces of distinctly different intensities.

The across-date differences in shadow profiles are depicted in Figures 8, 9 and 10 for different settlement types. The uncorrected images for the different acquisition dates $d_{1}$ and $d_{2}$ are denoted by $d_{1}$-image and $d_{2}$-image, and the shadow corrected images are given by $d_{1}$-corrected and $d_{2}$-corrected. The shadow correction has been performed with the refined algorithm and shadow masks detected with a fixed threshold of $0.25 Y_{\max }$.

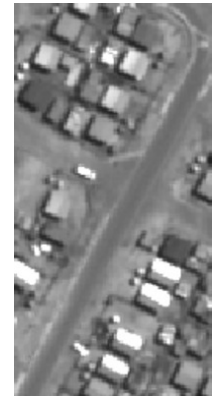

(a) $d_{1}$-image

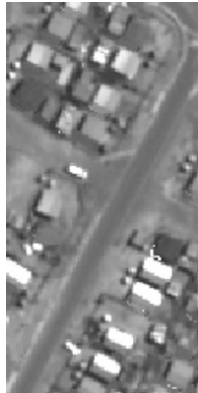

(b) $d_{1}$-corrected

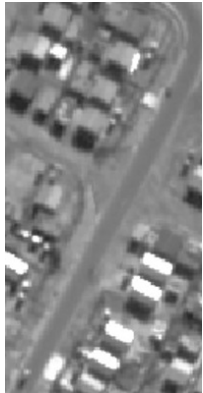

(c) $d_{2}$-image

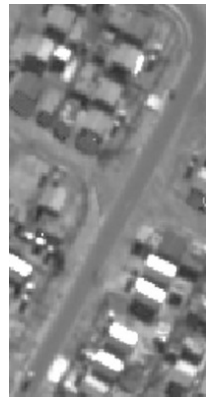

(d) $d_{2}$-corrected
Fig. 8. Formal settlement (FS) and its corresponding shadow corrected images for different acquisition dates.

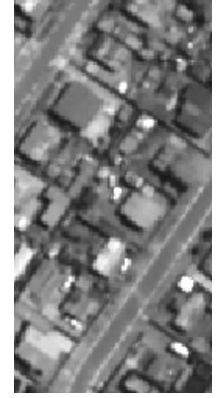

(a) $d_{1}$-image

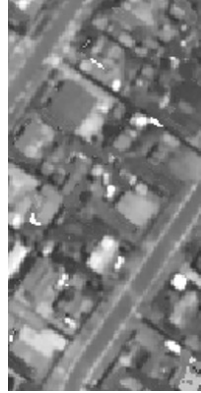

(b) $d_{1}$-corrected

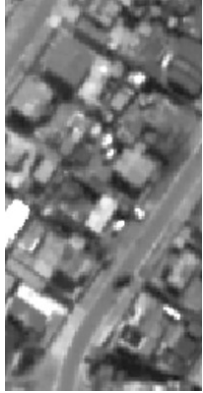

(c) $d_{2}$-image

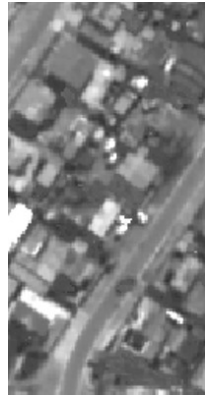

(d) $d_{2}$-corrected
Fig. 9. Formal settlement with backyard shacks (FSB) and its corresponding shadow corrected images for different acquisition dates.

\section{DATA DESCRIPTION}

Three prominent settlement types were considered as shown in Figures 8, 9 and 10, namely formal settlements (FS), formal settlements with backyard shacks (FSB) and ordered 


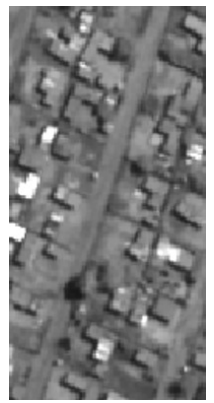

(a) $d_{1}$-image

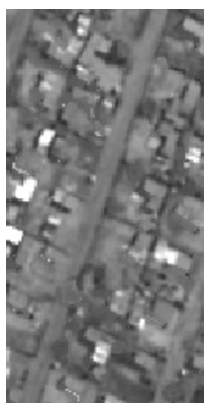

(b) $d_{1}$-corrected

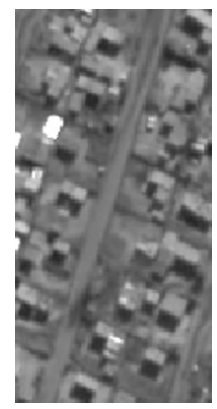

(c) $d_{2}$-image

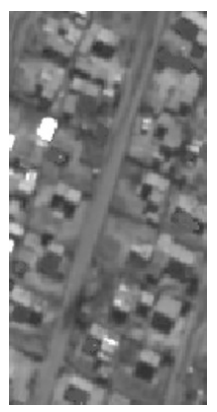

(d) $d_{2}$-corrected
Fig. 10. Ordered informal settlement (OIS) and its corresponding shadow corrected images for different acquisition dates.

informal settlements (OIS). Formal settlements have permanent residential structures positioned in a planned manner, while formal settlements with backyard shacks have residential structures accompanied by smaller shacks. Ordered informal settlements have permanent and semi-permanent residential structures ordered in a planned manner. In addition there is a non-builtup class (NBU) that includes natural vegetation, to test the separability between non-builtup areas and urban land-use types.

The study area is the $2.7 \times 9.3 \mathrm{~km}$ section of Soweto (Gauteng, South Africa), a subtropical highland of which two $0.6 \times 0.6 \mathrm{~m}$ resolution panchromatic QuickBird images were captured on 18 October $2005\left(d_{1}\right.$, early summer, rain season) and 30 May $2006\left(d_{2}\right.$, early winter). There are notable viewing- and illumination geometry differences between the two images, especially in the shadow profiles where $d_{2}$ exhibits longer shadows due to the Northern Hemisphere being inclined toward the Sun. Figure 11 shows the latest acquisition of the study area and the area selections of the land-use classes are indicated.

Representative polygon pairs of each settlement type were extracted in an assisted manner for both dates, most of which form spatially adjacent selections of the same settlement class for the purposes of creating separate training and testing sets. Training and testing data sets are denoted by $A$ and $B$, respectively, and the data sets are used interchangeably for either training or testing purposes.

The 11-bit panchromatic QuickBird scenes were converted to 8-bit images to enable visual inspection after each step and to simplify the texture feature calculations. The number of GLCM co-occurrence matrix entries is reduced by a factor of $2^{6}$ with this change in bit-depth, and LBP features remain largely unaffected since the intensity modification is a monotonic transformation. Across-date polygon pairs were then histogram matched and square image tiles with representative dimensions of $120 \times 120 \mathrm{~m}$ were obtained from every polygon.

\section{EXPERIMENTAL SETUP}

The purpose of the experiments in this study is to determine and compare the shadow detection accuracy of the various shadow detectors, and also to measure the change in settlement classification accuracy for the different shadow removal algorithms.

\section{A. Settlement classification accuracy}

A distinction is made between same-date and across-date experiments based on the hypothesis that shadow removal in the case of differing shadow profiles with across-date experiments will have a greater effect on settlement classification accuracy. For two-date experiments with area separation there are 4 possible same-date experiments and 8 different acrossdate experiments, as shown in Figure 12.

The scenery of a specific date is divided into two parts, namely areas $\mathrm{A}$ and $\mathrm{B}$, to provide separate training and testing data in order to reduce classifier overfitting. The area separation is performed in terms of whole polygon selections, which are depicted in Figure 11, with the aim of obtaining an approximately equal number of samples for both areas. The same area selections are used for images from other dates, so that an area is the identical set of polygon locations for all dates, but the actual image is then obtained from the specific date. Areas A and B are used interchangeably as either training or testing datasets to allow for more experiments to be performed.

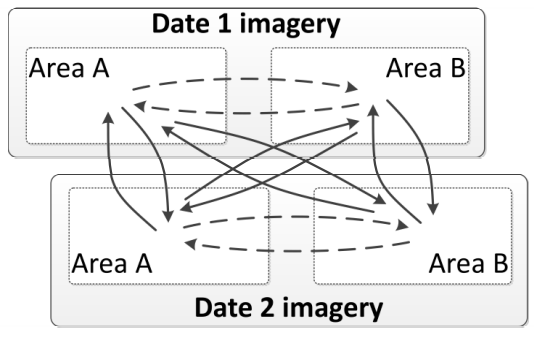

Fig. 12. The separation of same-date (--) and across-date (-) experiments.

Data sets consist of particular texture features and the corresponding ground truth settlement type classification associated with every extracted tile. The training data sets $\left(A_{d_{1}}\right.$ and $\left.A_{d_{2}}\right)$ have 55, 148, 70, and 133 tiles respectively for the FS, FSB, OIS and NBU settlement types. The testing data sets $\left(B_{d_{1}}\right.$ and $B_{d_{2}}$ ) contain 50, 120, 67, and 113 tiles for the FS, FSB, OIS and NBU classes, respectively. The training and testing data sets are also used respectively as testing and training data sets, while area separation between training and testing data sets is always maintained for same-date experiments.

Texture features are calculated per labelled tile, either using GLCM or LBP features. In the case of shadow masking the modified texture features are used where pixels falling inside the shadow masks are omitted during feature calculation. The first 13 of Haralick's GLCM features [16] are used with a window sized to the tile dimensions of $200 \times 200$ pixels. GLCM pairs are taken in all cardinal and ordinal directions with $\ell_{1}$-norms of 1 and 2 , respectively. The 10 basic patterns are used to calculate the LBP features [17] employing an 8point circle with radii of 1,4 and 8 to produce a total of 30 features.

A multi-layer perceptron is used as classifier with texture features as input and with the four discrete type classes FS, FSB, OIS and NBU as output. The number of input units of the perceptron is equal to the number of texture features (13 for GLCM and 30 for LBP), and the number of output units 


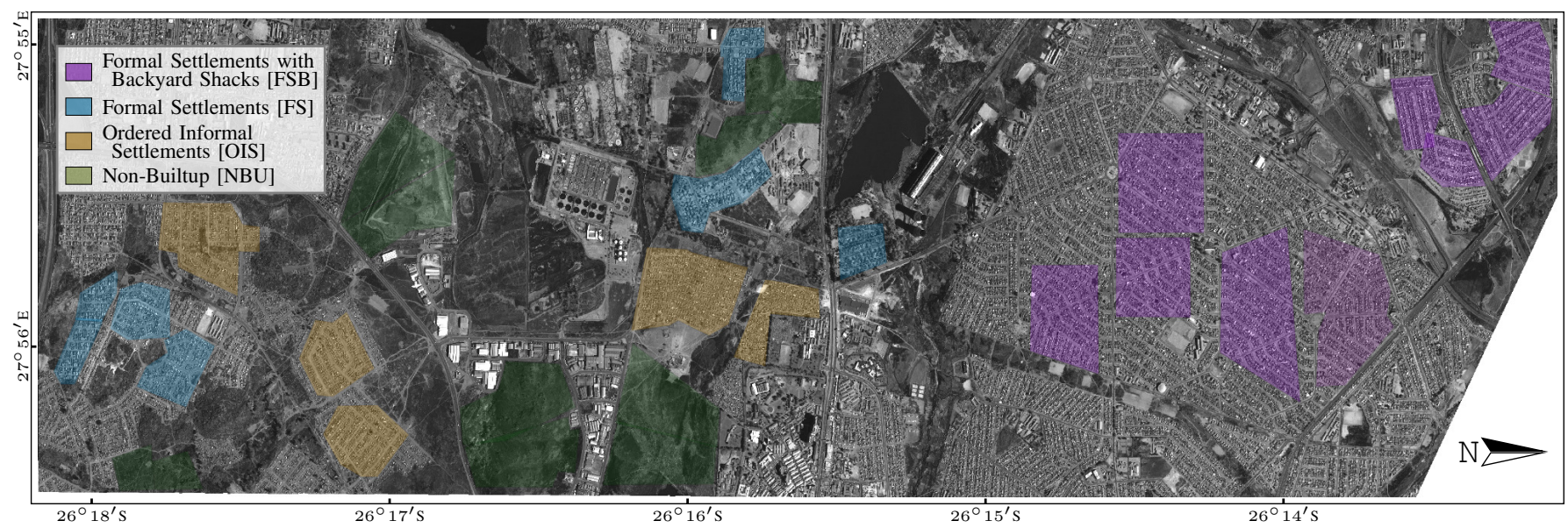

Fig. 11. The study area of Soweto, acquired on 30 May 2006, with polygon selections of the various land-use classes.

is determined by the number of classes, i.e. 4. The number of units in the single hidden layer is calculated as the sum of the number of attributes and classes, divided by two. The perceptron activation functions are unipolar sigmoids and for each experiment a momentum rate of 0.2 , learning rate of 0.3 and 500 training epochs are used.

For the same-date experiments a perceptron trained on one area will be tested on the other area of the same date. The 8 possible across-date experiments are depicted in Figure 12, where a perceptron trained on one area of a date will always be tested on an area of the other date. Every one of the 4 samedate and 8 across-date experiments is performed 10 times, with perceptron network weights initialized to a different random seed every time.

\section{B. Shadow detection accuracy}

The shadow detection accuracy is measured with the Jaccard similarity coefficient [40] (also known as the Jaccard index) using a representative sample of ground truth shadow masks over all settlement types for both dates. This similarity index measures the ratio of the intersected area to the area of the union between a detected shadow mask and the associated ground truth mask. The compared shadow masks are more similar if the Jaccard index is higher and a Jaccard index of 1 means that the compared shadow masks are exactly the same. The Jaccard index has been used in remote sensing research to measure the detection difference between change detectors [41], and unlike the Yule coefficient [42] it can measure absolute difference in the case of zero intersect.

The shadow detection accuracy is measured firstly to assess how similar a detected mask is to a human interpretation of the shadows via the ground truth shadow masks. The inherent lack of object distinction in panchromatic imagery, and the gradient presentation of shadows cause a substantial subjectivity even in the human treatment of shadow selection. This ambiguity that complicates all panchromatic shadow detection methods, means that there is no absolute ground truth, but the human-aided benchmark is set nonetheless to provide a point of reference. The detection accuracy is measured for every specific threshold value of the fixed threshold detector, as well as for the basic and supervised segmentation-based shadow detectors.

\section{RESUlTS AND Discussion}

The performance of the various shadow removal algorithms in improving settlement classification accuracy is given in this section. A statistical study is performed to determine shadow detection accuracy and its effect on settlement classification accuracy, and a discussion and interpretation of the results are delivered.

\section{A. Settlement classification accuracy}

The settlement classification accuracy with GLCM and LBP features are recorded for a range of threshold values used by a fixed thresholding shadow detector as shown in Figure 13. The mean accuracy of the 4 same-date experiments and the mean accuracy of the 8 across-date experiments are used in Figure 13, where every experiment accuracy is the mean accuracy of 10 repetitions, as explained in the previous section. Shadow masking, basic shadow correction and fine correction are the shadow removal algorithms paired with the fixed thresholding detector, and the same-date and across-date results are recorded separately.

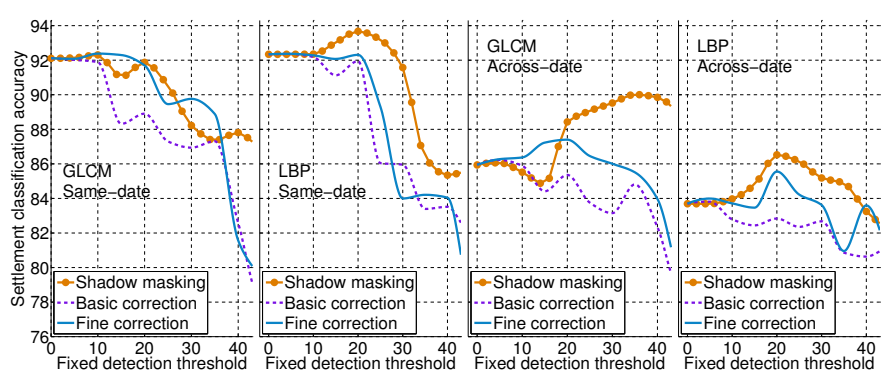

Fig. 13. Settlement classification accuracy comparison for fixed thresholding.

The case of no shadow removal occurs at a fixed threshold of 0 , and it is noted that the same-date experiments achieve a higher classification accuracy than the across-date experiments. Features calculated from the same-date scenery have 
greater similarity than across-date features, where viewingand illumination geometry distortions are introduced, so a reduced across-date classification accuracy is expected.

From Figure 13 it is clear that settlement classification accuracy benefits significantly more from across-date shadow removal than same-date shadow removal. Shadow masking in the case of LBP features has however improved samedate classification accuracy with statistical significance in 3 out of the 4 possible experiments at a fixed threshold of $0.2 Y_{\max }$. There was a statistically significant improvement in 7 out of the 8 across-date experiments with LBP and shadow masking, which decreased to 5 significant improvements when fine correction was used.

No significant same-date improvements were witnessed with GLCM, but in the across-date experiments the shadow masking and fine correction posted 5 and 3 statistically significant improvements, respectively. In Table I it is seen that GLCM improved the most with shadow masking to reach an accuracy of $90 \%(\kappa=0.86)$ at a fixed threshold of $0.35 Y_{\max }$. Of the fixed threshold methods the across-date settlement classification accuracy improvement with LBP was the greatest for shadow masking as well, where a value of $86.53 \%(\kappa=0.81)$ was attained at the threshold $0.2 Y_{\max }$.

TABLE I

SHADOW DETECTION AND SETTLEMENT CLASSIFICATION ACCURACIES

\begin{tabular}{|l|c|cc|cc|} 
& \multirow{2}{*}{$\begin{array}{c}\text { Jaccard } \\
\text { index }\end{array}$} & $\begin{array}{c}\text { Same- } \\
\text { date }\end{array}$ & $\begin{array}{c}\text { Across- } \\
\text { date }\end{array}$ & $\begin{array}{c}\text { Lame- } \\
\text { date }\end{array}$ & $\begin{array}{c}\text { Across- } \\
\text { date }\end{array}$ \\
\hline No removal & 0.00 & 92.11 & 85.94 & 92.34 & 83.69 \\
\hline Fixed masking & & 92.30 & $\mathbf{9 0 . 0 0}$ & 93.67 & 86.53 \\
Basic corr. & 0.45 & 92.12 & 86.23 & 92.36 & 83.81 \\
Fine corr. & & $\mathbf{9 2 . 3 8}$ & 87.40 & 92.37 & 85.56 \\
\hline Segmented & 0.29 & 90.87 & 88.02 & 93.28 & 86.11 \\
Supervised & 0.45 & 91.28 & 84.25 & $\mathbf{9 3 . 9 8}$ & $\mathbf{8 8 . 6 2}$ \\
\hline
\end{tabular}

The unsupervised segmentation-based shadow detector results are found in the 'Segmented' row in Table I, and the proposed supervised detector results in the 'Supervised' row. The basic segmentation-based detector achieved 5 and 4 statistically significant improvements out of 8 experiments in both of the across-date GLCM and LBP experiments with shadow masking, respectively. The proposed supervised segmentation-based detector with LBP features and shadow masking performed better than any of the fixed threshold shadow removal methods, with accuracies of $93.98 \%(\kappa=0.92)$ and $88.62 \%(\kappa=0.84)$ in the same-date and across-date experiments, respectively. Three of 4 same-date experiments and 7 out of 8 across-date experiments were improved with statistical significance in that case.

The supervised segmentation-based detector with GLCM features did not improve with shadow masking, however. This is likely due to the higher shadow omission error rate of supervised shadow detection. The irregular shadow detection omissions that result can influence all GLCM feature values while affecting only a small subset of LBP patterns when centered in shadow area, which may explain the discrepancy in performance between GLCM and LBP features. Additional shadow segment features may be added to reduce the omission rate of the supervised shadow detector, such as segment shape properties.

An across-date classification of the study area as imaged on $d_{1}$ was performed with supervised segmentation-based shadow removal and GLCM features, where the classifier was trained with all of the $d_{2}$ data. A mean accuracy of $89.1 \%$ was achieved after 10 experiments, of which a classification instance is depicted in Figure 14, which was obtained with majority voting from redundant tile cover of each polygon. The classification results are relatively accurate for the FSB, OIS and NBU classes when compared to Figure 11, but there is confusion between the FS class and the FSB and OIS classes primarily due to the small formal settlement training data size. There is also an underlying similarity between the FS and FSB classes that is hard to separate based on the texture features that were used.

Shadow differences are significantly aggravated in the case of taller buildings, and the shadow mask has the potential to occupy a relatively large portion of the image area. This might occur at lower solar elevations and shadow masking would then be a poor choice of shadow removal due to the large area that would have to be masked. Alternatively, shadow correction would retain all of the image area for texture calculation, at the expense of the inaccuracies caused by posterization.

Differing viewing angles may also cause significant changes in the texture of scenes with medium- and high-rise buildings, and paired with the shadow differences a loss in settlement classification accuracy is expected with texture features. The amplified anisotropy of land surfaces with tall buildings requires a specialized approach, but for the relatively flat texture of the considered settlement types the texture features perform well.

\section{B. Shadow detection accuracy}

The shadow detection accuracies of the fixed thresholding detector and the basic and supervised segmentation-based detectors were measured in terms of the Jaccard similarity coefficient with ground truth shadow masks. The fixed thresholding detector in its working threshold range as well as the proposed supervised detector improved the basic segmentation-based shadow detection accuracy from 0.29 to 0.45 as indicated in Table I.

Given that the basic segmentation-based detector in conjunction with shadow masking was able to improve the settlement classification accuracy in the across-date GLCM and LBP tests as well as in the same-date LBP tests, despite a significantly lower Jaccard index, indicates that this measure of shadow detection accuracy is a poor predictor of the settlement accuracy improvement.

Another interpretation of this result is that a significant increase in the similarity measure cannot clearly predict an equally significant increase in settlement classification accuracy, at least not for shadow detection algorithms operating in qualitatively different modes. The relationship between shadow detection accuracy and settlement classification accuracy has been more closely examined for the fixed threshold detector and the results are given in Figures 15 and 16. 


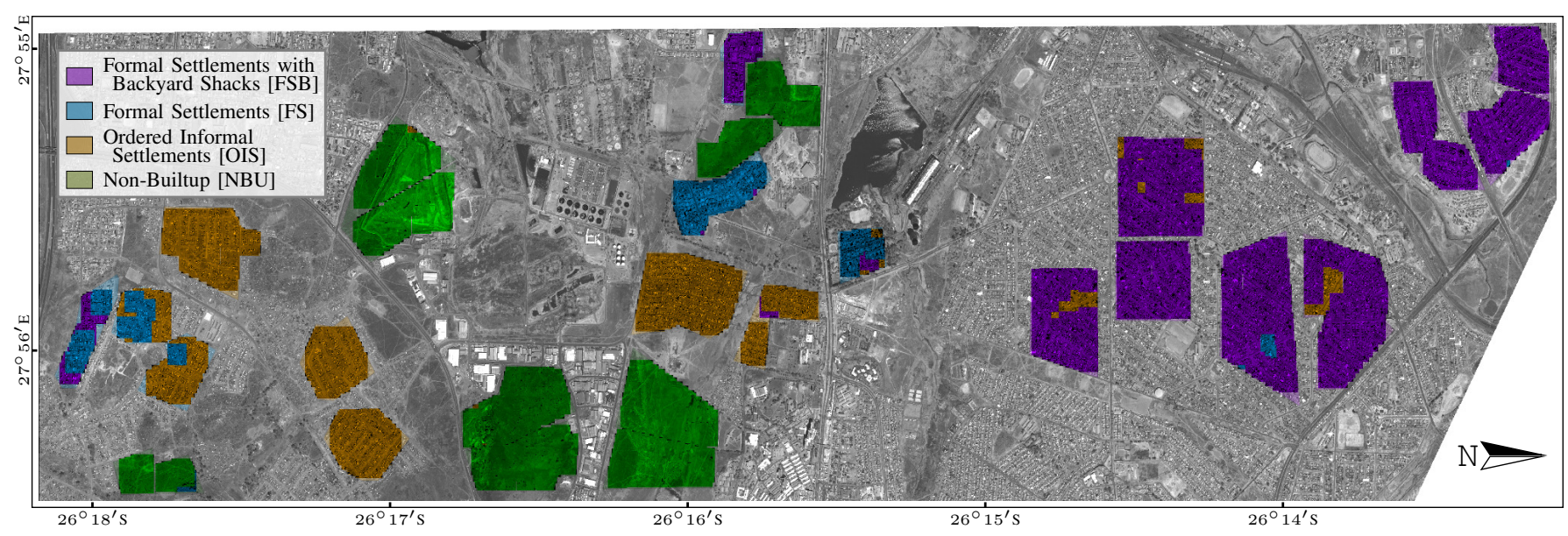

Fig. 14. A second image of the study area, acquired on 18 October 2005, with a thematic classification using training data from another date.

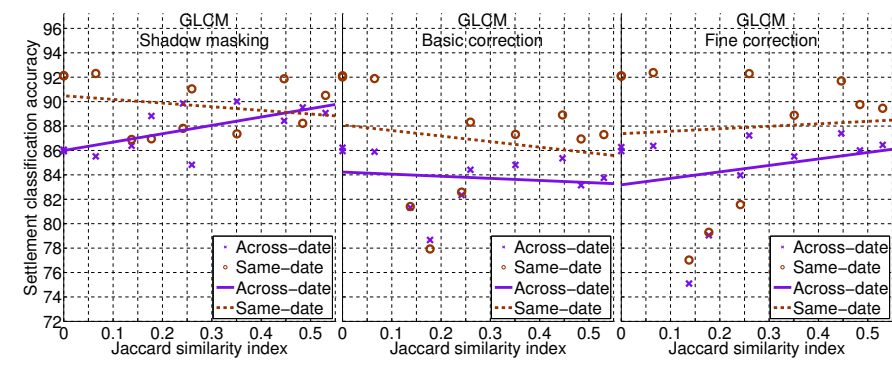

Fig. 15. The relationship between shadow detection accuracy and settlement classification accuracy for GLCM features.

To clearly discern the relationship between detection and classification accuracy the correlations of the same-date and across-date experiments are compared. For both the fixed threshold detection with GLCM and LBP experiments the same-date regression slopes are smaller for all shadow removal algorithms, as shown in Figures 15 and 16. This means that there is a stronger correlation for across-date experiments where a more accurate shadow mask improves settlement accuracy by a greater amount than would typically be seen in same-date improvements. It is expected that across-date experiments would benefit more from shadow removal and this is observed in the distinct difference between same-date and across-date correlations.

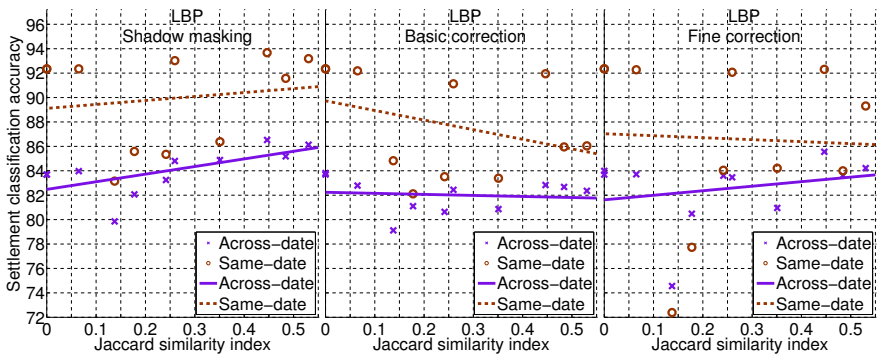

Fig. 16. The relationship between shadow detection accuracy and settlement classification accuracy for LBP features

The Pearson's correlation coefficients (denoted by $\rho$ ) of the same-date and across-date data points for fixed thresholding paired with the various shadow removal algorithms are shown in Table II. A Pearson's correlation coefficient measures the linear dependence between two variables and is defined as the covariance of the two variables divided by the product of their standard deviations. The correlation coefficient ranges from -1 to 1 , where a value of 1 implies that a linear equation can perfectly describe the relationship between the two variables. A linear relationship where one variable increases as another decreases is characterized by a correlation coefficient of -1 . When the value is 0 then there is no discernible linear relationship between the two variables.

The statistical significance of each correlation was also determined in terms of a p-value calculated using the Student's t-distribution for a transformation of the correlation. A pvalue is the probability of obtaining a test statistic at least as extreme as the one actually observed, assuming that the null hypothesis is true. The null hypothesis here is that there is no correlation between shadow detection accuracy and settlement classification accuracy, i.e. $\rho=0$, formulated with the aim of rejecting the null hypothesis to show that there is in fact correlation between the variables.

A p-value less than a small significance level of 0.05 would imply that the probability of observing a correlation given the null hypothesis is less than $5 \%$, which would constitute strong evidence against a zero correlation. In that case it can be stated that the observed correlation is probably not zero with statistical significance at a significance level of 0.05. Apart from the across-date shadow masking experiments showing statistically significant correlations at a significance level of 0.05 , the rest of the correlations are not significant due to the small number of samples.

With the emphasis placed on the null-hypothesis test of equal correlation for the same-date and across-date samples of a specific shadow removal algorithm, it is seen that there is a relatively small probability $\Delta$ p-val of obtaining correlation differences at least as extreme as were observed, given that the null-hypothesis is true. The $\Delta \mathrm{p}$-val is a notation used here to refer to the $\mathrm{p}$-value of the test statistic that measures the correlation difference between same-date and across-date experiments. The $\Delta \mathrm{p}$-val is calculated according to the cor- 
TABLE II

CORRELATION COEFFICIENTS AND STATISTICAL SIGNIFICANCE

\begin{tabular}{|c|c|c|c|c|c|}
\hline & & \multicolumn{2}{|c|}{ GLCM } & \multicolumn{2}{|c|}{ LBP } \\
\hline & & $\begin{array}{c}\text { Same- } \\
\text { date }\end{array}$ & $\begin{array}{c}\text { Across- } \\
\text { date }\end{array}$ & $\begin{array}{c}\text { Same- } \\
\text { date }\end{array}$ & $\begin{array}{c}\text { Across- } \\
\text { date }\end{array}$ \\
\hline \multirow{3}{*}{ Fixed Masking } & & -0.25 & 0.67 & 0.16 & 0.63 \\
\hline & p-val & 0.46 & 0.02 & 0.64 & 0.04 \\
\hline & $\Delta \mathrm{p}$-val & \multicolumn{2}{|c|}{0.05} & \multicolumn{2}{|c|}{0.02} \\
\hline \multirow{3}{*}{ Basic corr. } & $\rho$ & -0.19 & -0.14 & -0.36 & -0.11 \\
\hline & $\mathrm{p}$-val & 0.58 & 0.68 & 0.28 & 0.74 \\
\hline & $\Delta \mathrm{p}$-val & \multicolumn{2}{|c|}{0.29} & \multicolumn{2}{|c|}{0.14} \\
\hline \multirow{3}{*}{ Fine corr. } & $\rho$ & 0.06 & 0.26 & -0.04 & 0.23 \\
\hline & p-val & 0.84 & 0.44 & 0.90 & 0.49 \\
\hline & $\Delta \mathrm{p}$-val & \multicolumn{2}{|c|}{0.08} & \multicolumn{2}{|c|}{0.06} \\
\hline
\end{tabular}

related correlation coefficients case given by Meng et al. [43], where the Jaccard similarity coefficient is the shared variable between the same-date and across-date samples.

At a significance level of 0.1 it can be seen in Table II that the fixed thresholding masking and fine correction shadow removal algorithms for both GLCM and LBP features produced a significant correlation difference between same-date and across-date experiments. This supports the hypothesis that more accurate shadow removal produces greater improvements in settlement classification accuracy in the case of across-date experiments with notable shadow profile differences between the training and testing data sets.

\section{CONCLUSION}

Effective feature variance occurs during across-date settlement type classification due to differences in viewingand illumination geometry. The purpose of this study was to introduce calculated feature invariance by detecting and removing shadow differences that cause detrimental variation in texture features, and to test its efficacy in improving settlement type classification accuracy. An improved shadow detector was described as a segmentation-based algorithm with both unsupervised and supervised detection options, and it was shown that settlement type classification improved more than with a fixed threshold detector when LBP texture features were used.

The relationship between shadow detection accuracy and increases in settlement type classification accuracy was investigated experimentally and statistically. It was observed that there is a definite stronger trend with across-date classification where more accurate shadow removal resulted in a typically larger improvement in the measured settlement accuracy compared to same-date experiments. These results support the theory that it is the shadow removal specifically that improves classification accuracy, and that while increases in same-date accuracies were witnessed, the main benefit lies in across-date classification situations.

A minimal-supervised multitemporal classifier may then be trained on scenes from one date, and with the calculated feature invariance classify scenes from other dates with significantly improved accuracy when shadow removal is used. Future research may consider transductive classification and other domain adaptation methods, in order to compensate for the complex acquisition differences observed in remote sensing scenarios.

\section{ACKNOWLEDGMENTS}

The authors would like to thank the anonymous reviewers for their astute observations and keen advice. The financial assistance of the National Research Foundation (NRF) towards this research is hereby acknowledged. Opinions expressed and conclusions arrived at, are those of the authors and are not necessarily to be attributed to the NRF.

\section{REFERENCES}

[1] J. A. Voogt and T. R. Oke, "Effects of urban surface geometry on remotely-sensed surface temperature," International Journal of Remote Sensing, vol. 19, no. 5, pp. 895-920, 1998.

[2] F. Li, D. L. B. Jupp, and M. Thankappan, "Using high resolution DSM data to correct the terrain illumination effect in Landsat data," in MODSIM 2011 - 19th International Congress on Modelling and Simulation - Sustaining Our Future: Understanding and Living with Uncertainty, 2011, pp. 2402-2408.

[3] X. Wu, S. Collings, and P. Caccetta, "BRDF and illumination calibration for very high resolution imaging sensors," in Geoscience and Remote Sensing Symposium (IGARSS), 2010 IEEE International. IEEE, 2010, pp. 3162-3165.

[4] B. Huang, H. Zhang, and L. Yu, "Improving Landsat ETM+ urban area mapping via spatial and angular fusion with MISR multi-angle observations," Selected Topics in Applied Earth Observations and Remote Sensing, IEEE Journal of, vol. 5, no. 1, pp. 101-109, 2012.

[5] L. Abeigne-Ella, F. van den Bergh, B. J. van Wyk, and M. A. van Wyk, "A comparison of texture feature algorithms for urban settlement classification," in IEEE Geoscience and Remote Sensing Symposium (IGARSS 2008), vol. 3, July 2008, pp. 1308-1311.

[6] Y. Wei, Z. Zhao, and J. Song, "Urban building extraction from highresolution satellite panchromatic image using clustering and edge detection," in Geoscience and Remote Sensing Symposium, 2004. IGARSS'04. Proceedings. 2004 IEEE International, vol. 3, 2004, pp. 2008-2010.

[7] S. Wang and Y. Wang, "Shadow detection and compensation in high resolution satellite image based on retinex," in Image and Graphics, 2009. ICIG '09. Fifth International Conference on, Sept. 2009, pp. 209212.

[8] Ö. Aytekın, A. Erener, İ. Ulusoy, and Ş. Düzgün, "Unsupervised building detection in complex urban environments from multispectral satellite imagery," International Journal of Remote Sensing, vol. 33, no. 7, pp. 2152-2177, 2012.

[9] L. Lorenzi, F. Melgani, and G. Mercier, "A complete processing chain for shadow detection and reconstruction in VHR images," Geoscience and Remote Sensing, IEEE Transactions on, vol. 50, no. 9, pp. 34403452, 2012.

[10] X. Huang and L. Zhang, "Morphological building/shadow index for building extraction from high-resolution imagery over urban areas," Selected Topics in Applied Earth Observations and Remote Sensing, IEEE Journal of, vol. 5, no. 1, pp. 161-172, Feb. 2012.

[11] A. Makarau, R. Richter, R. Muller, and P. Reinartz, "Adaptive shadow detection using a blackbody radiator model," Geoscience and Remote Sensing, IEEE Transactions on, vol. 49, no. 6, pp. 2049-2059, June 2011.

[12] G. Tolt, M. Shimoni, and J. Ahlberg, "A shadow detection method for remote sensing images using VHR hyperspectral and LIDAR data," in Geoscience and Remote Sensing Symposium (IGARSS), 2011 IEEE International, July 2011, pp. 4423-4426.

[13] A. X. Falcão, P. A. V. Miranda, and A. Rocha, "A lineartime approach for image segmentation using graph-cut measures," in Proceedings of the 8th international conference on Advanced Concepts For Intelligent Vision Systems, ser. ACIVS'06. Berlin, Heidelberg: Springer-Verlag, 2006, pp. 138-149. [Online]. Available: http://dx.doi.org/10.1007/11864349_13

[14] W. Liu and F. Yamazaki, "Object-based shadow extraction and correction of high-resolution optical satellite images," Selected Topics in Applied Earth Observations and Remote Sensing, IEEE Journal of, vol. 5, no. 4, pp. 1296-1302, 2012.

[15] F. P. S. Luus, F. van den Bergh, and B. T. J. Maharaj, "The effects of shadow removal on across-date settlement type classification of QuickBird images," in Geoscience and Remote Sensing Symposium (IGARSS), 2012 IEEE International, July 2012, pp. 6196-6199. 
[16] R. M. Haralick, K. Shanmugam, and I. Dinstein, "Textural features for image classification," Systems, Man and Cybernetics, IEEE Transactions on, vol. 3, no. 6, pp. 610-621, Nov. 1973.

[17] T. Ojala, M. Pietikäinen, and T. Mäenpää, "Multiresolution gray-scale and rotation invariant texture classification with local binary patterns," Pattern Analysis and Machine Intelligence, IEEE Transactions on, vol. 24, no. 7, pp. 971-987, July 2002.

[18] F. van den Bergh, "The effects of viewing- and illumination geometry on settlement type classification of QuickBird images," in IEEE Geoscience and Remote Sensing Symposium (IGARSS 2011), July 2011, pp. 14251428.

[19] D. Ming, T. Ci, H. Cai, L. Li, C. Qiao, and J. Du, "Semivariogram-based spatial bandwidth selection for remote sensing image segmentation with mean-shift algorithm," Geoscience and Remote Sensing Letters, IEEE, vol. 9, no. 5, pp. 813-817, Sept. 2012.

[20] F. Moscheni, S. Bhattacharjee, and M. Kunt, "Spatio-temporal segmentation based on region merging," Pattern Analysis and Machine Intelligence, IEEE Transactions on, vol. 20, no. 9, pp. 897-915, Sept. 1998.

[21] R. Nock and F. Nielsen, "Statistical region merging," Pattern Analysis and Machine Intelligence, IEEE Transactions on, vol. 26, no. 11, pp. 1452-1458, Nov. 2004.

[22] F. Calderero and F. Marques, "Region merging techniques using information theory statistical measures," Image Processing, IEEE Transactions on, vol. 19, no. 6, pp. 1567-1586, June 2010.

[23] K. Haris, S. N. Efstratiadis, N. Maglaveras, and A. K. Katsaggelos, "Hybrid image segmentation using watersheds and fast region merging," Image Processing, IEEE Transactions on, vol. 7, no. 12, pp. 1684-1699, Dec. 1998.

[24] P. F. Felzenszwalb and D. P. Huttenlocher, "Efficient graph-based image segmentation," Int. J. Comput. Vision, vol. 59, no. 2, pp. 167-181, 2004. [Online]. Available: http://dx.doi.org/10.1023/B:VISI.0000022288.19776.77

[25] B. Peng, A. Xu, H. Li, and Y. Han, "Road extraction based on objectoriented from high-resolution remote sensing images," in Image and Data Fusion (ISIDF), 2011 International Symposium on. IEEE, 2011, pp. $1-4$.

[26] T. Sun, Z. Ren, and S. Ding, "Region-based semi-supervised clustering image segmentation," in Natural Computation (ICNC), 2011 Seventh International Conference on, vol. 4. IEEE, 2011, pp. 1855-1858.

[27] Z. Wu and R. Leahy, "An optimal graph theoretic approach to data clustering: theory and its application to image segmentation," Pattern Analysis and Machine Intelligence, IEEE Transactions on, vol. 15, no. 11, pp. 1101-1113, Nov. 1993.

[28] J. Shi and J. Malik, "Normalized cuts and image segmentation," Pattern Analysis and Machine Intelligence, IEEE Transactions on, vol. 22, no. 8, pp. 888-905, Aug. 2000.

[29] S. Wang and J. M. Siskind, "Image segmentation with ratio cut," Pattern Analysis and Machine Intelligence, IEEE Transactions on, vol. 25, no. 6, pp. 675-690, June 2003.

[30] B. Peng, L. Zhang, and D. Zhang, "Automatic image segmentation by dynamic region merging," Image Processing, IEEE Transactions on, vol. 20, no. 12, pp. 3592-3605, Dec. 2011.

[31] M. Baatz and A. Schäpe, "Multiresolution segmentation: An optimization approach for high quality multi-scale image segmentation," Angewandte Geographische Informations-Verarbeitung XII. Karlsruhe, pp. 12-23, 2000.

[32] Z. Hu, Z. Wu, Q. Zhang, Q. Fan, and J. Xu, "A spatially-constrained color-texture model for hierarchical vhr image segmentation," Geoscience and Remote Sensing Letters, IEEE, vol. 10, no. 1, pp. 120-124, Jan. 2013.

[33] D. Comaniciu and P. Meer, "Mean shift: a robust approach toward feature space analysis," Pattern Analysis and Machine Intelligence, IEEE Transactions on, vol. 24, no. 5, pp. 603-619, May 2002.

[34] X. Huang and L. Zhang, "An adaptive mean-shift analysis approach for object extraction and classification from urban hyperspectral imagery," Geoscience and Remote Sensing, IEEE Transactions on, vol. 46, no. 12, pp. 4173-4185, Dec. 2008.

[35] X. Li, H. Fan, Y. Zhao, and H. Zhang, "Graph cuts based image segmentation using local color and texture," in Proceedings - 4th International Congress on Image and Signal Processing, CISP 2011, vol. 3, 2011, pp. 1251-1255.

[36] C. M. Pun and N. Y. An, "Image segmentation using effective region merging strategy," International Journal of Digital Content Technology and its Applications (JDCTA), vol. 5, no. 8, pp. 59-69, 2011.

[37] J. Chen, M. Deng, P. Xiao, M. Yang, X. Mei, and H. Liu, "Objectoriented classification of high resolution imagery combining support vector machine with granular computing," Acta Geodaetica et Cartographica Sinica, vol. 40, no. 2, pp. 135-141+147, 2011.

[38] F. Meyer, "Topographic distance and watershed lines," Signal Processing, vol. 38, no. 1, pp. 113-125, 1994.

[39] Z. Zhang and F. Chen, "A shadow processing method of high spatial resolution remote sensing image," in Image and Signal Processing (CISP), 2010 3rd International Congress on, vol. 2, Oct. 2010, pp. 816820.

[40] P. Jaccard, "Étude comparative de la distribution florale dans une portion des Alpes et des Jura," Bulletin de la Société Vaudoise des Sciences Naturelles, vol. 37, pp. 547-579, 1901.

[41] M. İlsever, U. Altunkaya, and C. Ünsalan, "Pixel based change detection using an ensemble of fuzzy and binary logic operations," in Geoscience and Remote Sensing Symposium (IGARSS), 2012 IEEE International. IEEE, 2012, pp. 6185-6187.

[42] P. L. Rosin and E. Ioannidis, "Evaluation of global image thresholding for change detection," Pattern Recognition Letters, vol. 24, no. 14, pp. 2345-2356, 2003.

[43] X. Meng, R. Rosenthal, and D. Rubin, "Comparing correlated correlation coefficients," Psychological Bulletin, vol. 111, no. 1, pp. 172-175, January 1994.

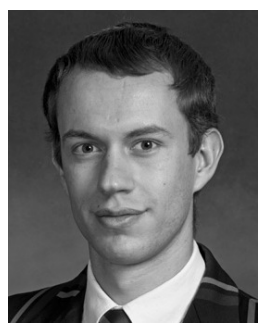

F.P.S. Luus received his B.Eng. and M.Eng. degrees from the University of Pretoria, South Africa, in 2007 and 2011, respectively. He is currently pursuing a Ph.D. degree in electronic engineering (machine learning) at the University of Pretoria, South Africa. His research interests include artificial intelligence, wireless communications and remote sensing.

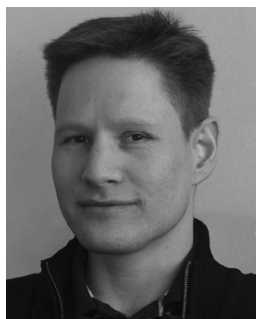

F. van den Bergh received the M.Sc. degree in computer science (machine vision) and the Ph.D. degree in computer science (particle swarm optimization) from the University of Pretoria, South Africa, in 2000 and 2002, respectively. He is currently a principal researcher at the Council for Scientific and Industrial Research. His research interests include automated feature extraction from high-resolution satellite images, as well as automated change detection. He maintains an active interest in particle swarm optimization and machine learning.

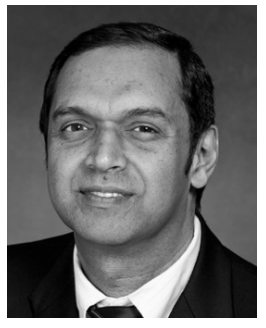

B.T.J. Maharaj received his M.Sc. Engineering from the University of Natal, M.Sc. in Operational Telecommunications from University of Coventry and Ph.D. in Electronic Engineering from University of Pretoria. He holds the SENTECH Chair in Broadband Wireless Multimedia Communications and is currently the Head of the Department in Electrical, Electronic and Computer Engineering at the University of Pretoria, South Africa. His research interests are in MIMO channel modeling, cognitive radio and optimization techniques. 